\title{
Chromium Enhances Insulin Responsiveness via AMPK
}

\author{
Nolan J. Hoffman ${ }^{1}$, Brent A. Penque ${ }^{1}$, Kirk M. Habegger ${ }^{2,3}$, Whitney Sealls ${ }^{1}$, Lixuan \\ Tackett $^{1}$, and Jeffrey S. Elmendorf ${ }^{1,2}$ \\ ${ }^{1}$ Department of Cellular and Integrative Physiology, Center for Diabetes Research, Indiana \\ University School of Medicine, Indianapolis, IN 46202 \\ ${ }^{2}$ Department of Biochemistry and Molecular Biology, Center for Diabetes Research, Indiana \\ University School of Medicine, Indianapolis, IN 46202 \\ ${ }^{3}$ Department of Medicine - Endocrinology, Diabetes \& and Metabolism University of Alabama at \\ Birmingham
}

\begin{abstract}
Trivalent chromium $\left(\mathrm{Cr}^{3+}\right)$ is known to improve glucose homeostasis. $\mathrm{Cr}^{3+}$ has been shown to improve plasma membrane-based aspects of glucose transporter GLUT4 regulation and increase activity of the cellular energy sensor $5^{\prime}$ AMP-activated protein kinase (AMPK). However, the mechanism(s) by which $\mathrm{Cr}^{3+}$ improves insulin responsiveness and whether AMPK mediates this action is not known. In this study we tested if $\mathrm{Cr}^{3+}$ protected against physiological hyperinsulinemia-induced plasma membrane cholesterol accumulation, cortical filamentous actin (F-actin) loss and insulin resistance in L6 skeletal muscle myotubes. In addition, we performed mechanistic studies to test our hypothesis that AMPK mediates the effects of $\mathrm{Cr}^{3+}$ on GLUT4 and glucose transport regulation. Hyperinsulinemia-induced insulin-resistant L6 myotubes displayed excess membrane cholesterol and diminished cortical F-actin essential for effective glucose transport regulation. These membrane and cytoskeletal abnormalities were associated with defects in insulin-stimulated GLUT4 translocation and glucose transport. Supplementing the culture medium with pharmacologically relevant doses of $\mathrm{Cr}^{3+}$ in the picolinate form $(\mathrm{CrPic})$ protected against membrane cholesterol accumulation, F-actin loss, GLUT4 dysregulation and glucose transport dysfunction. Insulin signaling was neither impaired by hyperinsulinemic conditions nor enhanced by $\mathrm{CrPic}$, whereas CrPic increased AMPK signaling. Mechanistically, siRNA-mediated depletion of AMPK abolished the protective effects of CrPic against GLUT4 and glucose transport dysregulation. Together these findings suggest that the micronutrient $\mathrm{Cr}^{3+}$, via increasing AMPK activity, positively impacts skeletal muscle cell insulin sensitivity and glucose transport regulation.
\end{abstract}

(C) 2014 Elsevier Inc. All rights reserved.

Corresponding Author: Jeffrey S. Elmendorf, PhD, Department of Cellular and Integrative Physiology, Indiana University School of Medicine, VanNuys Medical Science Building Rm. 307, Indianapolis, IN 46202, TEL: 317-274-7852, FAX: 317-274-3318, jelmendo@iupui.edu.

Disclosure Summary: The authors have nothing to disclose.

Publisher's Disclaimer: This is a PDF file of an unedited manuscript that has been accepted for publication. As a service to our customers we are providing this early version of the manuscript. The manuscript will undergo copyediting, typesetting, and review of the resulting proof before it is published in its final citable form. Please note that during the production process errors may be discovered which could affect the content, and all legal disclaimers that apply to the journal pertain. 


\section{Keywords}

AMP-activated protein kinase; Cholesterol; Chromium; GLUT4; Insulin

\section{INTRODUCTION}

A review of randomized controlled trials analyzing the effects of trivalent chromium $\left(\mathrm{Cr}^{3+}\right)$ supplementation on glucose metabolism found that $\mathrm{Cr}^{3+}$ supplementation significantly improved glycemic control among patients with type 2 diabetes [1]. During the course of our studies to identify and dissect the mechanism(s) by which $\mathrm{Cr}^{3+}$ improves glycemic status, we found that $\mathrm{Cr}^{3+}$ enhances glucose transporter GLUT4 translocation and glucose transport in 3T3-L1 adipocytes via lowering plasma membrane cholesterol [2-3].

Building on these in vitro findings, analyses of glucose-intolerant humans and animal models revealed increased skeletal muscle membrane cholesterol and reduced cortical filamentous actin (F-actin) [4]. Studies using 3T3-L1 adipocytes and L6 myotubes suggest that key metabolic derangements (e.g., hyperinsulinemia, hyperlipidemia) known to accelerate the worsening of insulin resistance and progression to type 2 diabetes increase plasma membrane cholesterol [4-6]. In these insulin-resistant cell model systems this gain in plasma membrane cholesterol was found to compromise cortical F-actin structure essential for GLUT4 and glucose transport regulation [4-6]. Further investigation demonstrated that actin cytoskeletal dynamics are an essential feature of insulin-stimulated glucose transport in intact skeletal muscle [7-8], which is responsible for approximately $80 \%$ of postprandial glucose disposal [9] and a major peripheral site of insulin resistance [10]. However, the impact of $\mathrm{Cr}^{3+}$ supplementation on membrane cholesterol, cortical F-actin and glucose transport regulation in skeletal muscle cells is not known. Interestingly, in vitro and in vivo studies have reported that $\mathrm{Cr}^{3+}$ treatment increases $5^{\prime}$-AMP activated protein kinase (AMPK) activity [2, 11-15]. Given that AMPK phosphorylates and inhibits 3-hydroxy-3methyl-glutaryl coenzyme A reductase (HMGR), the rate-limiting enzyme in cholesterol synthesis, $\mathrm{Cr}^{3+}$ could potentially mediate its beneficial effects on GLUT4 and glucose transport regulation via AMPK activation.

Currently, $\mathrm{Cr}^{3+}$ can be found in several nutritional supplements including $\mathrm{Cr}^{3+}$ picolinate (CrPic). CrPic, the most popular form of $\mathrm{Cr}^{3+}$, represents the second overall highest-selling nutritional supplement only behind calcium supplements [16]. Interestingly, CrPic effectiveness in 3T3-L1 adipocytes has been demonstrated to be dependent on the diabetic milieu [3], similar to the observation that CrPic supplementation does not affect glycemic status in people without diabetes [1]. What is not known is whether CrPic supplementation can protect against membrane cholesterol accumulation and F-actin loss in the insulinresistant state, and whether these beneficial aspects of $\mathrm{CrPic}$ action can prevent the development of insulin resistance in skeletal muscle cells. Here we report that $\mathrm{CrPic}$ supplementation in vitro prevents L6 skeletal muscle myotube membrane cholesterol accumulation, cortical F-actin loss and glucose transport dysfunction induced by physiological hyperinsulinemia. These studies were paired with mechanistic investigation to 
determine whether AMPK mediates the beneficial effects of CrPic on GLUT4 and glucose transport regulation.

\section{RESULTS}

\section{CrPic protects against hyperinsulinemia-induced GLUT4/glucose transport dysfunction}

Studies first evaluated if $\mathrm{CrPic}$ protects against physiological hyperinsulinemia-induced glucose transport dysfunction in L6 myotubes. Our CrPic treatment parameters were selected based on our in vitro data showing that exposing 3T3-L1 adipocytes to CrPic, ranging from $10 \mathrm{nM}$ to $10 \mu \mathrm{M}$, for $16 \mathrm{~h}$ lowered plasma membrane cholesterol and enhanced glucose transport [2]. Given that the lower $10-100 \mathrm{nM}$ dose of CrPic is closely akin to concentrations expected to be observed in tissues from individuals supplemented with $\mathrm{CrPic}$ in vivo [2], we incubated L6 myotubes in the absence or presence of $100 \mathrm{nM} \mathrm{CrPic}$ for a total of $16 \mathrm{~h}$ with or without $5 \mathrm{nM}$ insulin for the last $12 \mathrm{~h}$. Acute insulin-stimulation increased glucose transport by $97 \%$ in control, non-CrPic treated cells (Fig. 1A, compare bars 1 and 2), and this was inhibited by $67 \%$ in myotubes exposed to hyperinsulinemia with no CrPic (Fig. 1A, compare bars 2 and 4). No change in basal glucose transport levels were observed (Fig. 1A, compare bars 1 and 3). This hyperinsulinemia-induced glucose transport dysfunction was not observed in CrPic-treated myotubes (Fig. 1A, compare bars 2 and 8). A similar insulin-stimulated GLUT4 translocation defect and protective effect of $\mathrm{CrPic}$ was observed (Fig. 1B, compare bars 2, 4 and 8). Basal and acute insulin-stimulated glucose transport and GLUT4 translocation in control cells were not affected by CrPic (Figs. 1A and 1B, compare bars 1 and 5,2 and 6).

\section{Membrane and cytoskeletal defects do not occur in the presence of CrPic}

Recent evidence has suggested that AMPK agonists protect against hyperinsulinemiainduced GLUT4 dysregulation in L6 myotubes [17]. This beneficial effect of AMPK activity was shown to involve protection against excess membrane cholesterol accumulation and cortical F-actin loss [17]. Similarly, we find here that insulin-induced insulin-resistant L6 myotubes display a slight increase $(20 \%, \mathrm{p}<0.05)$ and decrease $(11 \%, \mathrm{p}<0.05)$ in membrane cholesterol and cortical F-actin, respectively (Figs. 2A and 2B, compare bars 1 and 2). In contrast, these hyperinsulinemia-induced changes were not seen in myotubes cultured in the presence of CrPic (Figs. 2A and 2B, compare bars 1, 2, and 4). No significant effect on membrane cholesterol or F-actin was seen in control myotubes exposed to $\mathrm{CrPic}$ alone (Figs. $2 \mathrm{~A}$ and $2 \mathrm{~B}$, compare bars 1 and 3 ).

\section{Insulin signal transduction is not impaired by hyperinsulinemia nor enhanced by CrPic}

Studies have documented that neither physiological hyperinsulinemia nor effects of CrPic are attributed to decreases or increases, respectively, in insulin signaling [2-3, 8, 18], although some studies have suggested that CrPic amplifies insulin signaling [19-21]. Therefore, key signaling parameters of insulin action were evaluated. As previously reported in this insulin-resistant L6 myotube model system [8], basal or insulin-stimulated phosphorylation of Akt2 at serine residue 474 (Ser474) was not impaired (Fig. 3A, compare lanes/bars 1-4). Phosphorylation of the Akt substrate of $160 \mathrm{kDa}$ (AS160)/TBC1 domain family, member 4 (TBC1D4), which will be referred to in all subsequent text and figures as 
AS160, was detected using a phospho-Akt substrate antibody and was also not impaired (Fig. 3B, compare lanes/bars 1-4). In addition, the presence of CrPic in the medium did not affect basal or insulin-stimulated phosphorylation of Akt2 Ser474 or AS160 under control or hyperinsulinemic conditions (Figs. 3A and 3B, lanes/bars 5-8).

\section{CrPic increases AMPK activity and AMPK loss prevents CrPic's beneficial action}

Consistent with previous observations in $\mathrm{Cr}^{3+}$-treated cells [2, 11-13] and tissues from $\mathrm{Cr}^{3+}$ supplemented animals [14-15], myotubes cultured in either control or hyperinsulinemic conditions showed a CrPic-stimulated increase in AMPK activity, as evidenced by increased phosphorylation of the catalytic alpha subunit at Threonine residue 172 (Thr172) (Fig. 4A). Chronic insulin exposure did not affect Thr172 phosphorylation (Fig. 4A, compare lanes/ bars 1 and 3). In addition, CrPic-stimulated Thr172 phosphorylation trended (*p=0.06) to be greater in hyperinsulinemia-induced insulin-resistant myotubes compared to control myotubes (Fig. 4A, compare lanes/bars 2 and 4).

To confirm that CrPic treatment of L6 myotubes resulted in increased AMPK activity, the phosphorylation of acetyl CoA carboxylase (ACC) at Serine residue 79 (Ser79), a well characterized substrate of AMPK, was evaluated. A 17\% increase in phosphorylation of ACC at Ser79 in myotubes treated with CrPic compared to control (Fig. 4B, compare lanes/ bars 1 and 2) was detected, similarly to the increase in AMPK Thr172 phosphorylation in the presence of CrPic. Hyperinsulinemia had no effect on ACC Ser79 (Fig. 4B, compare lanes/bars 1 and 3). In line with AMPK Thr172 findings, CrPic also induced a significant increase in phosphorylation of ACC at Ser79 in myotubes exposed to hyperinsulinemia that was significantly enhanced compared to control myotubes (Fig. 4B, compare lanes/bars 2 and 4). An antibody that recognizes HMGR when phosphorylated at Serine residue 872 was also tested to determine if CrPic-induced AMPK activation was associated with an increase in phosphorylation of HMGR Ser872, yet the intensity of the bands detected were too faint for accurate analysis.

To determine if AMPK activation was required for CrPic action, we next tested the effect of siRNA-mediated knockdown of the catalytic a subunits of AMPK (siAMPK). Since L6 myotubes express $a 1$ and a 2 AMPK subunits, oligonucleotides against both were used simultaneously for knockdown of AMPK catalytic activity. The combination of a 1 and a 2 isoform-specific oligonucleotides reduced the detectable pan-AMPKa protein by $75 \%$ (Fig. 5A). Increased AMPK phosphorylation was observed in control and hyperinsulinemic myotubes transfected with scrambled control (siScramble) oligonucleotides and treated with CrPic (Fig. 5B, lanes/bars 1-4). Although the CrPic-mediated increase in AMPK phosphorylation did not reach statistical significance in siScramble myotubes, this trend was apparent. In addition, we observed a characteristic AMPK activation by the AMPK agonist 5-aminoimidazole-4-carboxamide-1-beta-D-ribonucleoside (AICAR) in siScramble myotubes (data not shown), as we have previously reported in these cells using this knockdown system [17]. Consistent with the substantial loss of AMPK in siAMPK myotubes, the stimulatory effect of CrPic and AICAR on AMPK Thr172 phosphorylation in these cells was abolished under both control and hyperinsulinemic conditions (Fig. 5B, lanes/bars 6, 8, and 9). The reduction in AMPKa did not impair basal and insulin-stimulated 
insulin receptor signaling (data not shown) or signaling to Akt2 Ser474 in any of the treatment groups (Fig 5C). Mechanistically, the protective effects of CrPic on insulinstimulated GLUT4 translocation and glucose transport observed in hyperinsulinemiainduced insulin-resistant siScramble myotubes (Figs. 6A and 6B, compare bars 2 and 4) were not observed in siAMPK myotubes (Figs. 6A and 6B, compare bars 6 and 8).

\section{DISCUSSION}

Skeletal muscle from insulin-resistant animal models and humans display excess membrane cholesterol that diminishes F-actin structure and GLUT4/glucose transport regulation by insulin [4]. This pathologic cholesterol accumulation was also evident in this study and was associated with a loss of cortical F-actin and GLUT4 regulation in insulin-resistant L6 myotubes. Previous studies with skeletal muscle isolated from obese, insulin-resistant Zucker rats demonstrated (via methyl- $\beta$-cyclodextrin-mediated removal of cholesterol) that excess membrane cholesterol and decreased F-actin impair insulin-stimulated glucose transport [17]. With new data supporting that key derangements of the prediabetic milieu (e.g., hyperinsulinemia, hyperlipidemia) provoke a cholesterolgenic response via increasing hexosamine biosynthesis activity, plasma membrane cholesterol accumulation may represent a previously unrecognized stress contributing to the development of skeletal muscle insulin resistance and acceleration to type 2 diabetes [4-6].

Therapeutically, we found that CrPic positively impacts membrane cholesterol and cortical F-actin levels important for normal GLUT4 and glucose transport regulation by insulin in vitro. We also did not observe any effect of CrPic on Akt or AS160 activation, consistent with our previous studies in 3T3-L1 demonstrating that the regulation of GLUT4 translocation by $\mathrm{CrPic}$ did not involve known insulin signaling proteins such as the insulin receptor, insulin receptor substrate-1, phosphatidylinositol 3-kinase, and Akt [2]. A caveat here is that we did not evaluate the phosphorylation of TBC1 domain family, member 1 (TBC1D1), a TBC1 family member functionally involved in contraction- and AMPKstimulated rather than insulin-stimulated GLUT4 translocation in skeletal muscle [22-25]. Although it is possible that TBC1D1 is a target of CrPic, AS160 is the primary GLUT4containing vesicle Rab-GAP implicated in insulin-stimulated GLUT4 translocation (as reviewed in [26]) and has also been shown to be phosphorylated by AMPK [27].

Our studies utilizing siRNA-targeted knockdown of AMPK catalytic activity are consistent with AMPK mediating the protective effect of CrPic against GLUT4 and glucose transport dysregulation. Unfortunately, due to the large amount of siRNA oligos and cellular starting material required for membrane cholesterol and F-actin analyses in siScramble and siAMPK myotubes, these analyses were not performed in the present study. Nevertheless, in support of this membrane cholesterol mechanism of $\mathrm{Cr}^{3+} /$ AMPK action, we have found that ATPindependent (i.e., AICAR conversion to the $5^{\prime}$-AMP analogue ZMP) and ATP-dependent (i.e., 2,4-Dinitrophenol (DNP)-induced mitochondrial uncoupling that prevents cellular production of ATP) mechanisms of AMPK activation lowered membrane cholesterol in L6 myotubes. The cholesterol-lowering action of AICAR was also observed to protect against insulin-induced membrane cholesterol accumulation, F-actin loss and insulin resistance [17]. Moreover, the beneficial effect of these AMPK-activating agents on enhancing the insulin- 
stimulated increase in membrane GLUT4 was eliminated by replenishing the reduced membrane cholesterol back to levels observed in control cells. Intriguingly, this did not suppress the insulin mimetic stimulation of GLUT4 translocation by AICAR and DNP; whereas siAMPK knockdown did, suggesting that increased AMPK activity affects basal and insulin-stimulated GLUT4 regulation by divergent mechanisms. As we previously reported in 3T3-L1 adipocytes [2], the present study showed that CrPic, unlike AICAR and DNP, did not increase functional plasma membrane GLUT4 content or glucose transport in the absence of insulin. Perhaps this is due to the slight activation of AMPK observed in insulin-sensitive cells that may not be sufficient to stimulate GLUT4 translocation and glucose transport. Whereas the greater increase in AMPK activity seen in insulin-resistant cells may not signal directly to GLUT4, per se, it may rather suppress excess cholesterol biosynthesis that then positively impacts GLUT4 regulation by insulin. Because AMPK activation can occur rapidly, for example in response to cellular energy stress or stimulation by an AMPK agonist, we tested shorter CrPic treatment times in L6 myotubes. We observed increased phosphorylation of AMPK by $100 \mathrm{nM}$ CrPic only after overnight incubations, thus we used the 16-h treatment period in the present study (see Supplemental Fig. 1). Previous studies in 3T3-L1 adipocytes demonstrated similar increases in phosphorylation of AMPK following overnight incubations with $10 \mathrm{nM}-1000 \mathrm{nM}$ CrPic [2, 11]. Similarly, other studies have shown that long-term $24 \mathrm{~h}$ treatment with $10 \mathrm{nM}$ CrPic increases levels of phosphorylated AMPK and ACC [12]. Consistent with the protective effects of overnight CrPic treatment observed in this study, previous studies in 3T3-L1 adipocytes have shown that CrPic treatment increases GLUT4 translocation to the PM [2-3, 28-29]. Studies have suggested that CrPic treatment in skeletal muscle cells can also increase GLUT4 transcription [30]. Although it is possible that increased GLUT4 transcription contributed to increased GLUT4 translocation, our previous study in 3T3-L1 adipocytes did not observe any affect of CrPic on total GLUT4 protein levels in crude membrane fractions [2].

As yet, we have a limited understanding about the temporal relationship and basis of AMPK activation by CrPic, and this deserves further experimental dissection. As reviewed in [31], there exist several signaling pathways that modify Thr172 phosphorylation within the activation loop of the AMPK a-subunit kinase domain, which is essential for kinase activity. The level of phosphorylation at this site is exquisitely regulated by AMP and ADP levels, both due to stimulation of phosphorylation by upstream kinases and inhibition of dephosphorylation by the protein phosphatase PP2A, both effects being antagonized by high concentrations of ATP. These effects are entirely substrate mediated; i.e., they are due to binding of the nucleotides to AMPK and not to the upstream kinase or protein phosphatase. AMPK is only active after phosphorylation of the critical Thr172 residue within the kinase domain by upstream kinases. The major upstream kinase is a complex between the tumor suppressor LKB1 and two accessory subunits, STRAD and MO25. The calmodulindependent protein kinase kinases (CaMKK)s, especially CaMKK $\beta$, can also act as alternate upstream kinases phosphorylating Thr172. This latter effect is triggered by a rise in cytosolic calcium without requiring an increase in AMP.

The fact that AMPK is a key sensor of cellular fuel and energy status is of interest in the context of CrPic-responsiveness, as it appears that the diabetic milieu, and thus cellular 
energy status, greatly influences CrPic action similarly to that of AMPK. Perhaps the baseline fuel/energy status determines AMPK's responsiveness to CrPic. Interestingly, data suggest that one potential mechanism by which metformin enhances insulin action is by increasing membrane fluidity [32-33]. As we have observed after CrPic treatment, metformin has also been reported to increase GLUT4 translocation [34-36]. It has also been shown that the relative enhancing effect of metformin is higher in myotubes incubated in 25 $\mathrm{mM}$ glucose rather than in $5 \mathrm{mM}$ glucose, consistent with its selective action in hyperglycemic conditions in vivo [37]. We have observed that the relative enhancing effect of CrPic on GLUT4 translocation is also higher in 3T3-L1 adipocytes incubated in $25 \mathrm{mM}$ glucose rather than $5 \mathrm{mM}$ glucose [3]. An increase in plasma membrane cholesterol was noted in adipocytes cultured in $25 \mathrm{mM}$ glucose, and the beneficial action of $\mathrm{CrPic}$ was attributed to lowering plasma membrane cholesterol content to levels measured in control, insulin-sensitive adipocytes cultured in $5 \mathrm{mM}$ glucose [3]. As reported for metformin [3841], the effects of CrPic are not attributed to increased expression of GLUT4 protein, but rather its translocation and/or activation state [2, 42-44]. In line with these observations, our studies herein suggest a shared mechanism for the beneficial effects of metformin and CrPic on cellular insulin sensitivity. Although intermittent study has coupled membrane fluidity to insulin sensitivity [45-47], a mechanistic understanding has remained elusive. It is an interesting concept that plasma membrane cholesterol lowering may be a common mechanism of action of $\mathrm{CrPic}$ and metformin, as well as several other antidiabetic agents known to display AMPK-stimulating and cholesterol-lowering properties, e.g. berberine [48-50], cryptotanshinone [51] and fibrates [52-53]. Moreover, skeletal muscle contraction and exercise signaling may enhance insulin sensitivity via AMPK-mediated suppression of cholesterol synthesis.

In summary, the present study suggests that AMPK activation is an important aspect of the mechanism of $\mathrm{Cr}^{3+}$ action in insulin-resistant skeletal muscle cells. Furthermore, this study demonstrates that membrane cholesterol influences cytoskeletal structure essential for insulin-regulated GLUT4 translocation and glucose transport. Although speculative, we view these findings as a possible indication that hyperinsulinemia-induced membrane and cytoskeletal defects in skeletal muscle could negatively impinge on GLUT4 regulation, perhaps before defects are induced in the insulin signaling machinery (as discussed in [26]). The present study, using an in vitro model of hyperinsulinemia that resembles the pathophysiological insulin concentrations observed in prediabetic patients, provides evidence that $\mathrm{CrPic}$ improves insulin action by protecting against pathological increases in membrane cholesterol that compromise F-actin integrity required for proper insulinregulated GLUT4 translocation. While these findings are noteworthy, future studies are warranted to test the effects of $\mathrm{CrPic}$ against the complex in vivo milieu (i.e., secreted factors and cytokines, altered circulating/intramyocellular lipid profile, etc.) reflecting the pathophysiology of prediabetes. It is interesting that many clinical studies involving $\mathrm{Cr}^{3+}$ supplementation have been performed in patients already diagnosed with type 2 diabetes. Therefore, the effects of $\mathrm{Cr}^{3+}$ in these patients may have been less if other defects, which may not be amendable to $\mathrm{Cr}^{3+}$ therapy, are already present. In addition, many of these studies were performed in patients already receiving antidiabetic and cardiovascular therapies such as metformin and statins that may mask the mechanism of $\mathrm{Cr}^{3+}$ action. Future 
animal and human $\mathrm{Cr}^{3+}$ supplementation studies are required to determine if the cholesterollowering action of $\mathrm{Cr}^{3+}$ and increased AMPK activity in skeletal muscle could potentially delay and/or prevent the onset of insulin resistance and type 2 diabetes.

\section{METHODS AND MATERIALS}

\section{Cell culture and treatments}

Rat L6 muscle cells stably expressing GLUT4 that carries an exofacial myc-epitope (L6GLUT4myc; generously obtained from Dr. Amira Klip, (The Hospital for Sick Children, Toronto, Canada) were cultured as previously described [8]. Myoblasts were maintained in a-Minimum essential medium (a-MEM) containing $5.5 \mathrm{mM}$ glucose (Gibco, Grand Island, NY) and 10\% fetal bovine serum (FBS; HyClone Laboratories, Grand Island, NY), and differentiated into multinucleated myotubes with $2 \%$ FBS. All studies used myotubes between 4 and 6 days post-initiation of differentiation. Differentiated cells were cultured in control medium in the absence or presence of $100 \mathrm{nM} \mathrm{CrPic}$ (Nutrition 21, Purchase, NY) for $16 \mathrm{~h}$. Induction of insulin-induced insulin resistance was performed as previously described [8] by treating the cells with $5 \mathrm{nM}$ insulin for $12 \mathrm{~h}$. Note that induction of insulininduced insulin resistance began $4 \mathrm{~h}$ after the initiation of CrPic exposure. Cells were serum starved for $30 \mathrm{~min}$ before all experiments. During the final $20 \mathrm{~min}$ (or $5 \mathrm{~min}$ for insulin signaling analyses) of this serum starvation, cells were left untreated or acutely stimulated with $100 \mathrm{nM}$ insulin. Note longer serum starvation periods we tried did not enhance the characteristic low response (1.5-2.0 fold) of this cell line to insulin.

\section{Immunocytochemistry}

GLUT4myc and F-actin labeling was performed as previously described [8]. Briefly, myotubes were fixed with $2 \%$ paraformaldehyde/PBS. After fixation, cells were either left unpermeabilized (GLUT4myc) or were permeabilized (F-actin) for $15 \mathrm{~min}$ at room temperature in $0.2 \%$ Triton X-100/PBS. Cells were then blocked for $1 \mathrm{~h}$ at room temperature in Odyssey Blocking Buffer (LI-COR Biosciences, Lincoln, NE). Samples were incubated in a 1:50 dilution of primary antibody overnight at $4{ }^{\circ} \mathrm{C}$, followed by incubation at room temperature with infrared-conjugated secondary antibody for $1 \mathrm{~h}$. Images were collected and quantified with the LI-COR Odyssey imaging system as previously described [8]. Immunofluorescent intensity was normalized to intensity from Syto60, a fluorescent nucleic acid stain (Life Technologies, Grand Island, NY).

\section{Membrane and cholesterol analyses}

A plasma membrane-enriched fraction from cultured cells was prepared as described by Khayat et al. [54]. Briefly, myotube monolayers grown on 10-cm-diameter dishes were gently scraped with a rubber policeman in $5 \mathrm{ml}$ of ice-cold HES homogenization buffer (in mM: 250 sucrose, 20 HEPES, 2 EGTA and $3 \mathrm{NaN}_{3}$, pH 7.4) containing freshly added protease inhibitors (in $\mu \mathrm{M}$ : $200 \mathrm{PMSF}, 1$ leupeptin and 1 pepstatin A) and homogenized through a 22-gauge needle 10 times. The homogenate was centrifuged at $760 \mathrm{~g}$ for $5 \mathrm{~min}$ at $4{ }^{\circ} \mathrm{C}$, and the resultant supernatant was centrifuged at $31,000 \mathrm{~g}$ for $20 \mathrm{~min}$ to separate a plasma membrane-enriched pellet from an intracellular microsome supernatant. The plasma membrane pellet was resuspended in HES buffer and assayed for protein (Bradford) and 
cholesterol (Amplex Red) content as previously described. Several unpublished analyses we have performed revealed that measured changes in plasma membrane cholesterol were similarly reflected in total cellular membrane fractions prepared by centrifuging the original cellular homogenate at 5,000 $\mathrm{g}$ for $20 \mathrm{~min}$ at $4^{\circ} \mathrm{C}$ and then subjecting the supernatant to centrifugation at $100,000 \mathrm{~g}$ for $30 \mathrm{~min}$. As the number of 10 -cm-diameter dishes required to obtain this total membrane fraction were half that required to prepare plasma membraneenriched fractions, we obtained total membrane fractions in the current study.

\section{2-deoxy-D-glucose transport assays}

Following treatments, cells were incubated in glucose-free buffer $(125 \mathrm{mM} \mathrm{NaCl}, 5 \mathrm{mM}$ $\mathrm{KCl}, 1.8 \mathrm{mM} \mathrm{CaCl}_{2}, 2.6 \mathrm{mM} \mathrm{MgSO}_{4}, 25 \mathrm{mM}$ HEPES, $2 \mathrm{mM}$ pyruvate and 2\% BSA) for 30 $\mathrm{min}$, then either left in the basal state or stimulated with $100 \mathrm{nM}$ insulin for $20 \mathrm{~min}$. Glucose uptake was initiated with the addition of 2-deoxy-[1,2- $\left.{ }^{3} \mathrm{H}\right]$-D-glucose $(0.055 \mu \mathrm{Ci} / \mu \mathrm{l})$.

Nonspecific uptake was quantitated via cell-associated radioactivity in the presence of 20 $\mu \mathrm{M}$ cytochalasin $\mathrm{B}$. After $5 \mathrm{~min}$, uptake was terminated via four quick washes with ice-cold PBS. Cells were solubilized in $1 \mathrm{~N} \mathrm{NaOH}$ and $\left[{ }^{3} \mathrm{H}\right]$ was measured by liquid scintillation. Counts were normalized to total cellular protein, as determined by the Bradford method.

\section{SDS-PAGE analyses}

Total cell extracts were prepared from 10-cm-diameter dishes of L6 myotubes. Myotubes were washed two times with ice-cold PBS and scraped or homogenized using a Polytron, respectively, in $1 \mathrm{ml}$ lysis buffer ( $25 \mathrm{mM}$ Tris, $\mathrm{pH}$ 7.4, $50 \mathrm{mM} \mathrm{NaF}, 10 \mathrm{mM} \mathrm{Na}_{3} \mathrm{P}_{2} \mathrm{O}_{7}, 137$ $\mathrm{mM} \mathrm{NaCl}, 10 \%$ glycerol and $1 \%$ Nonidet P-40) containing $2 \mathrm{mM}$ PMSF, $2 \mathrm{mM} \mathrm{Na}_{3} \mathrm{VO}_{4}, 5$ $\mu \mathrm{g} / \mathrm{ml}$ aprotinin, $10 \mu \mathrm{M}$ leupeptin and $1 \mu \mathrm{M}$ pepstatin A then rotated for $15 \mathrm{~min}$ at $4^{\circ} \mathrm{C}$. Insoluble material was separated from the soluble extract by centrifugation for $15 \mathrm{~min}$ at $4^{\circ} \mathrm{C}$. Protein concentrations were determined via the Bradford method and equivalent protein amounts were separated by 7.5\% SDS-PAGE. The resolved fractions were transferred to nitrocellulose (Bio-Rad, Hercules, CA). Phospho-Akt-2 and total Akt-2 were detected with anti-phospho-Akt-2 (Ser474) (Genscript, Piscataway, NJ) and anti-Akt-2 (Cell Signaling Technology, Danvers, MA) antibodies. We also used phosphospecific antibodies to AMPK Thr172 and AS160 (Cell Signaling Technology, Danvers, MA) and to HMGR Ser872 and ACC Ser79 (Millipore, Temecula, CA). Equal protein loading was confirmed by Ponceau staining and by immunoblot analysis with anti-ACC and anti-AMPK (Cell Signaling Technology, Danvers, MA), and anti-Actin antibody (Cytoskeleton, Denver, CO). All immunoblots were labeled with IR-conjugated secondary antibodies and analyzed via the Odyssey imaging system (LI-COR Biosciences, Lincoln, NE).

\section{siRNA design and transfection}

Three independent oligonuceotide sequences, designed and purchased from Ambion (Austin, TX), were tested for each of the two alpha subunit isoforms. The oligonucleotides with the highest knockdown efficiency for a 1 and a 2 were respectively: CGA GUU GAC UGG ACAUAA Att (siRNA ID\#: 194424) and GCA ACU AUC AAA GAC AUA Ctt (siRNA ID\#: 194794). As these cells express both alpha isoforms, the combination of the two nucleotides led to the greatest knockdown efficiency. Ambion's Negative Control \#1 
siRNA (Cat \#:4635) was used as a control in all experiments. For all knockdown experiments cells were seeded as described before. Cells were first transfected at approximately $48 \mathrm{~h}$ post seeding (or $\sim 60 \%$ confluency). Calcium phosphate transfection protocol was utilized as follows, 60 pmol of siRNA was added to siRNA mix: $15 \mu \mathrm{ddd}_{2} \mathrm{O}$, $15 \mu$ Buffer A ( $0.5 \mathrm{M} \mathrm{CaCl}_{2}, 0.1 \mathrm{M}$ HEPES (pH 7.0)) and $30 \mu$ Buffer B $(0.28 \mathrm{M} \mathrm{NaCl}$, $0.75 \mathrm{mM} \mathrm{NaH}_{2} \mathrm{PO}_{4}, 0.75 \mathrm{mM} \mathrm{Na}_{2} \mathrm{HPO}_{4}$ and $0.05 \mathrm{M}$ HEPES (pH 7.0)) and after $10 \mathrm{~min}$ at room temperature, the siRNA mix was added to each well of a 12 well plate containing 600 $\mu \mathrm{l}$ DMEM + 5\% FBS and incubated 12-16 h. Following 12-16 h incubation, media was aspirated and replaced with DMEM $+2 \%$ FBS. Additional transfection was repeated $72 \mathrm{~h}$ after initial transfection. Cells were treated and assayed $72 \mathrm{~h}$ after final transfection.

\section{Statistics}

Values are presented as means \pm SEM. The significance of differences between means was evaluated by ANOVA. Where differences among groups were indicated, the Newman-Keuls test was used for post hoc comparison between groups. Statistical comparisons of the percent change of cholesterol, F-actin, pAMPK/AMPK, and pACC/ACC from control were performed by a one-sample, two-tailed Student's $t$ test. GraphPad Prism 5 software (La Jolla, CA) was used for all analyses. $P<0.05$ was considered significant.

\section{Supplementary Material}

Refer to Web version on PubMed Central for supplementary material.

\section{Acknowledgments}

Grants/Fellowships: This work was supported by the National Institutes of Health AT001846, DK082773 and DK082773-01S1 (JSE), the Indiana University Diabetes and Obesity Research Training Program DeVault Fellowship (NJH, KMH) and grant T32-DK064466 (NJH), the Moenkhaus Award (BAP), and the Indiana Center for Vascular Biology and Medicine HL079995 (WS).

We are grateful to Drs. Amira Klip and Steve Waters for generously providing the GLUT4myc expressing L6 myotubes and L6 myotube protocols. We thank Nutrition 21 for providing the CrPic.

\section{References}

1. Balk EM, Tatsioni A, Lichtenstein AH, Lau J, Pittas AG. Effect of chromium supplementation on glucose metabolism and lipids: a systematic review of randomized controlled trials. Diabetes Care. 2007; 30:2154-63. [PubMed: 17519436]

2. Chen G, Liu P, Pattar GR, Tackett L, Bhonagiri P, Strawbridge AB, et al. Chromium activates glucose transporter 4 trafficking and enhances insulin-stimulated glucose transport in 3T3-L1 adipocytes via a cholesterol-dependent mechanism. Mol Endocrinol. 2006; 20:857-70. [PubMed: 16339278]

3. Pattar GR, Tackett L, Liu P, Elmendorf JS. Chromium picolinate positively influences the glucose transporter system via affecting cholesterol homeostasis in adipocytes cultured under hyperglycemic diabetic conditions. Mutat Res. 2006; 610:93-100. [PubMed: 16870493]

4. Habegger KM, Penque BA, Sealls W, Tackett L, Bell LN, Blue EK, et al. Fat-induced membrane cholesterol accrual provokes cortical filamentous actin destabilisation and glucose transport dysfunction in skeletal muscle. Diabetologia. 2012; 55:457-67. [PubMed: 22002007]

5. Bhonagiri P, Pattar GR, Habegger KM, McCarthy AM, Tackett L, Elmendorf JS. Evidence coupling increased hexosamine biosynthesis pathway activity to membrane cholesterol toxicity and cortical 
filamentous actin derangement contributing to cellular insulin resistance. Endocrinology. 2011; 152:3373-84. [PubMed: 21712361]

6. Penque BA, Hoggatt AM, Herring BP, Elmendorf JS. Hexosamine Biosynthesis Impairs Insulin Action via a Cholesterolgenic Response. Mol Endocrinol. 2013; 27:536-47. [PubMed: 23315940]

7. Brozinick JT Jr, Hawkins ED, Strawbridge AB, Elmendorf JS. Disruption of cortical actin in skeletal muscle demonstrates an essential role of the cytoskeleton in glucose transporter 4 translocation in insulin-sensitive tissues. J Biol Chem. 2004; 279:40699-706. [PubMed: 15247264]

8. McCarthy AM, Spisak KO, Brozinick JT, Elmendorf JS. Loss of cortical actin filaments in insulinresistant skeletal muscle cells impairs GLUT4 vesicle trafficking and glucose transport. Am J Physiol Cell Physiol. 2006; 291:C860-8. [PubMed: 16774991]

9. Ferrannini E, Smith JD, Cobelli C, Toffolo G, Pilo A, DeFronzo RA. Effect of insulin on the distribution and disposition of glucose in man. J Clin Invest. 1985; 76:357-64. [PubMed: 3894421]

10. DeFronzo RA, Gunnarsson R, Bjorkman O, Olsson M, Wahren J. Effects of insulin on peripheral and splanchnic glucose metabolism in noninsulin-dependent (type II) diabetes mellitus. J Clin Invest. 1985; 76:149-55. [PubMed: 3894418]

11. Sealls W, Penque BA, Elmendorf JS. Evidence that chromium modulates cellular cholesterol homeostasis and ABCA1 functionality impaired by hyperinsulinemia--brief report. Arterioscler Thromb Vasc Biol. 2011; 31:1139-40. [PubMed: 21311039]

12. Wang YQ, Dong Y, Yao MH. Chromium picolinate inhibits resistin secretion in insulin-resistant 3T3-L1 adipocytes via activation of amp-activated protein kinase. Clin Exp Pharmacol Physiol. 2009; 36:843-9. [PubMed: 19298540]

13. Zhao P, Wang J, Ma H, Xiao Y, He L, Tong C, et al. A newly synthetic chromium complexchromium (D-phenylalanine)3 activates AMP-activated protein kinase and stimulates glucose transport. Biochem Pharmacol. 2009; 77:1002-10. [PubMed: 19073152]

14. Penumathsa SV, Thirunavukkarasu M, Samuel SM, Zhan L, Maulik G, Bagchi M, et al. Niacin bound chromium treatment induces myocardial Glut-4 translocation and caveolar interaction via Akt, AMPK and eNOS phosphorylation in streptozotocin induced diabetic rats after ischemiareperfusion injury. Biochim Biophys Acta. 2009; 1792:39-48. [PubMed: 19027847]

15. Thirunavukkarasu M, Penumathsa SV, Juhasz B, Zhan L, Cordis G, Altaf E, et al. Niacin-bound chromium enhances myocardial protection from ischemia-reperfusion injury. Am J Physiol Heart Circ Physiol. 2006; 291:H820-6. [PubMed: 16840737]

16. Vinson JA. So many choices, so what's a consumer to do?: A commentary on "Effect of chromium niacinate and chromium picolinate supplementation on lipid peroxidation, TNF-alpha, IL-6, CRP, glycated hemoglobin, triglycerides, and cholesterol levels in blood of streptozotocin-treated diabetic rats". Free Radic Biol Med. 2007; 43:1121-3. [PubMed: 17854707]

17. Habegger KM, Hoffman NJ, Ridenour CM, Brozinick JT, Elmendorf JS. AMPK enhances insulinstimulated GLUT4 regulation via lowering membrane cholesterol. Endocrinology. 2012; 153:2130-41. [PubMed: 22434076]

18. Chen G, Raman P, Bhonagiri P, Strawbridge AB, Pattar GR, Elmendorf JS. Protective effect of phosphatidylinositol 4,5-bisphosphate against cortical filamentous actin loss and insulin resistance induced by sustained exposure of 3T3-L1 adipocytes to insulin. J Biol Chem. 2004; 279:39705-9. [PubMed: 15277534]

19. Wang ZQ, Zhang XH, Russell JC, Hulver M, Cefalu WT. Chromium picolinate enhances skeletal muscle cellular insulin signaling in vivo in obese, insulin-resistant JCR:LA-cp rats. J Nutr. 2006; 136:415-20. [PubMed: 16424121]

20. Brautigan DL, Kruszewski A, Wang H. Chromium and vanadate combination increases insulininduced glucose uptake by 3T3-L1 adipocytes. Biochem Biophys Res Commun. 2006; 347:769_ 73. [PubMed: 16842748]

21. Wang H, Kruszewski A, Brautigan DL. Cellular chromium enhances activation of insulin receptor kinase. Biochemistry. 2005; 44:8167-75. [PubMed: 15924436]

22. Ducommun S, Wang HY, Sakamoto K, MacKintosh C, Chen S. Thr649Ala-AS160 knock-in mutation does not impair contraction/AICAR-induced glucose transport in mouse muscle. Am J Physiol Endocrinol Metab. 2012; 302:E1036-43. [PubMed: 22318952] 
23. Chen S, Murphy J, Toth R, Campbell DG, Morrice NA, Mackintosh C. Complementary regulation of TBC1D1 and AS160 by growth factors, insulin and AMPK activators. Biochem J. 2008; 409:449-59. [PubMed: 17995453]

24. Pehmoller C, Treebak JT, Birk JB, Chen S, Mackintosh C, Hardie DG, et al. Genetic disruption of AMPK signaling abolishes both contraction- and insulin-stimulated TBC1D1 phosphorylation and 14-3-3 binding in mouse skeletal muscle. Am J Physiol Endocrinol Metab. 2009; 297:E665-75. [PubMed: 19531644]

25. Szekeres F, Chadt A, Tom RZ, Deshmukh AS, Chibalin AV, Bjornholm M, et al. The RabGTPase-activating protein TBC1D1 regulates skeletal muscle glucose metabolism. Am J Physiol Endocrinol Metab. 2012; 303:E524-33. [PubMed: 22693207]

26. Hoffman NJ, Elmendorf JS. Signaling, cytoskeletal and membrane mechanisms regulating GLUT4 exocytosis. Trends Endocrinol Metab. 2011; 22:110-6. [PubMed: 21216617]

27. Treebak JT, Taylor EB, Witczak CA, An D, Toyoda T, Koh HJ, et al. Identification of a novel phosphorylation site on TBC1D4 regulated by AMP-activated protein kinase in skeletal muscle. Am J Physiol Cell Physiol. 2010; 298:C377-85. [PubMed: 19923418]

28. Wang YQ, Yao MH. Effects of chromium picolinate on glucose uptake in insulin-resistant 3T3-L1 adipocytes involve activation of p38 MAPK. J Nutr Biochem. 2009; 20:982-91. [PubMed: 19195868]

29. Wang Y, Van Oort MM, Yao M, Van der Horst DJ, Rodenburg KW. Insulin and chromium picolinate induce translocation of CD36 to the plasma membrane through different signaling pathways in 3T3-L1 adipocytes, and with a differential functionality of the CD36. Biol Trace Elem Res. 2011; 142:735-47. [PubMed: 20721637]

30. Qiao W, Peng Z, Wang Z, Wei J, Zhou A. Chromium improves glucose uptake and metabolism through upregulating the mRNA levels of IR, GLUT4, GS, and UCP3 in skeletal muscle cells. Biol Trace Elem Res. 2009; 131:133-42. [PubMed: 19283340]

31. Hardie DG, Sakamoto K. AMPK: a key sensor of fuel and energy status in skeletal muscle. Physiology (Bethesda). 2006; 21:48-60. [PubMed: 16443822]

32. Wiernsperger NF. Membrane physiology as a basis for the cellular effects of metformin in insulin resistance and diabetes. Diabetes Metab. 1999; 25:110-27. [PubMed: 10443322]

33. Muller S, Denet S, Candiloros H, Barrois R, Wiernsperger N, Donner M, et al. Action of metformin on erythrocyte membrane fluidity in vitro and in vivo. Eur J Pharmacol. 1997; 337:103-10. [PubMed: 9389387]

34. Matthaei S, Reibold JP, Hamann A, Benecke H, Haring HU, Greten H, et al. In vivo metformin treatment ameliorates insulin resistance: evidence for potentiation of insulin-induced translocation and increased functional activity of glucose transporters in obese (fa/fa) Zucker rat adipocytes. Endocrinology. 1993; 133:304-11. [PubMed: 8391425]

35. Fischer Y, Thomas J, Rosen P, Kammermeier H. Action of metformin on glucose transport and glucose transporter GLUT1 and GLUT4 in heart muscle cells from healthy and diabetic rats. Endocrinology. 1995; 136:412-20. [PubMed: 7835271]

36. Hundal HS, Ramlal T, Reyes R, Leiter LA, Klip A. Cellular mechanism of metformin action involves glucose transporter translocation from an intracellular pool to the plasma membrane in L6 muscle cells. Endocrinology. 1992; 131:1165-73. [PubMed: 1505458]

37. Klip A, Guma A, Ramlal T, Bilan PJ, Lam L, Leiter LA. Stimulation of hexose transport by metformin in L6 muscle cells in culture. Endocrinology. 1992; 130:2535-44. [PubMed: 1572281]

38. Ciaraldi TP, Kong AP, Chu NV, Kim DD, Baxi S, Loviscach M, et al. Regulation of glucose transport and insulin signaling by troglitazone or metformin in adipose tissue of type 2 diabetic subjects. Diabetes. 2002; 51:30-6. [PubMed: 11756319]

39. Thomas CR, Turner SL, Jefferson WH, Bailey CJ. Prevention of dexamethasone-induced insulin resistance by metformin. Biochem Pharmacol. 1998; 56:1145-50. [PubMed: 9802324]

40. Handberg A, Kayser L, Hoyer PE, Voldstedlund M, Hansen HP, Vinten J. Metformin ameliorates diabetes but does not normalize the decreased GLUT 4 content in skeletal muscle of obese (fa/fa) Zucker rats. Diabetologia. 1993; 36:481-6. [PubMed: 8335168] 
41. Rouru J, Koulu M, Peltonen J, Santti E, Hanninen V, Pesonen U, et al. Effects of metformin treatment on glucose transporter proteins in subcellular fractions of skeletal muscle in (fa/fa) Zucker rats. Br J Pharmacol. 1995; 115:1182-7. [PubMed: 7582542]

42. Cefalu WT, Wang ZQ, Zhang XH, Baldor LC, Russell JC. Oral chromium picolinate improves carbohydrate and lipid metabolism and enhances skeletal muscle Glut-4 translocation in obese, hyperinsulinemic (JCR-LA corpulent) rats. J Nutr. 2002; 132:1107-14. [PubMed: 12042418]

43. Morris B, Gray T, MacNeil S. Evidence for chromium acting as an essential trace element in insulin-dependent glucose uptake in cultured mouse myotubes. J Endocrinol. 1995; 144:135-41. [PubMed: 7891015]

44. Goto Y, Kida K. Insulin-like action of chromate on glucose transport in isolated rat adipocytes. Jpn J Pharmacol. 1995; 67:365-8. [PubMed: 7650869]

45. Czech MP. Insulin action and the regulation of hexose transport. Diabetes. 1980; 29:399-409. [PubMed: 6991331]

46. Pilch PF, Thompson PA, Czech MP. Coordinate modulation of D-glucose transport activity and bilayer fluidity in plasma membranes derived from control and insulin-treated adipocytes. Proc Natl Acad Sci U S A. 1980; 77:915-8. [PubMed: 6987672]

47. Whitesell RR, Regen DM, Beth AH, Pelletier DK, Abumrad NA. Activation energy of the slowest step in the glucose carrier cycle: break at 23 degrees $C$ and correlation with membrane lipid fluidity. Biochemistry. 1989; 28:5618-25. [PubMed: 2775725]

48. Tang LQ, Wei W, Chen LM, Liu S. Effects of berberine on diabetes induced by alloxan and a high-fat/high-cholesterol diet in rats. J Ethnopharmacol. 2006; 108:109-15. [PubMed: 16759828]

49. Turner N, Li JY, Gosby A, To SW, Cheng Z, Miyoshi H, et al. Berberine and its more biologically available derivative, dihydroberberine, inhibit mitochondrial respiratory complex I: a mechanism for the action of berberine to activate AMP-activated protein kinase and improve insulin action. Diabetes. 2008; 57:1414-8. [PubMed: 18285556]

50. Lee YS, Kim WS, Kim KH, Yoon MJ, Cho HJ, Shen Y, et al. Berberine, a natural plant product, activates AMP-activated protein kinase with beneficial metabolic effects in diabetic and insulinresistant states. Diabetes. 2006; 55:2256-64. [PubMed: 16873688]

51. Kim EJ, Jung SN, Son KH, Kim SR, Ha TY, Park MG, et al. Antidiabetes and antiobesity effect of cryptotanshinone via activation of AMP-activated protein kinase. Mol Pharmacol. 2007; 72:62-72. [PubMed: 17429005]

52. Han SH, Quon MJ, Koh KK. Beneficial vascular and metabolic effects of peroxisome proliferatoractivated receptor-alpha activators. Hypertension. 2005; 46:1086-92. [PubMed: 16230515]

53. Ferre P. The biology of peroxisome proliferator-activated receptors: relationship with lipid metabolism and insulin sensitivity. Diabetes. 2004; 53 (Suppl 1):S43-50. [PubMed: 14749265]

54. Khayat ZA, Tsakiridis T, Ueyama A, Somwar R, Ebina Y, Klip A. Rapid stimulation of glucose transport by mitochondrial uncoupling depends in part on cytosolic $\mathrm{Ca} 2+$ and cPKC. Am J Physiol. 1998; 275:C1487-97. [PubMed: 9843710] 


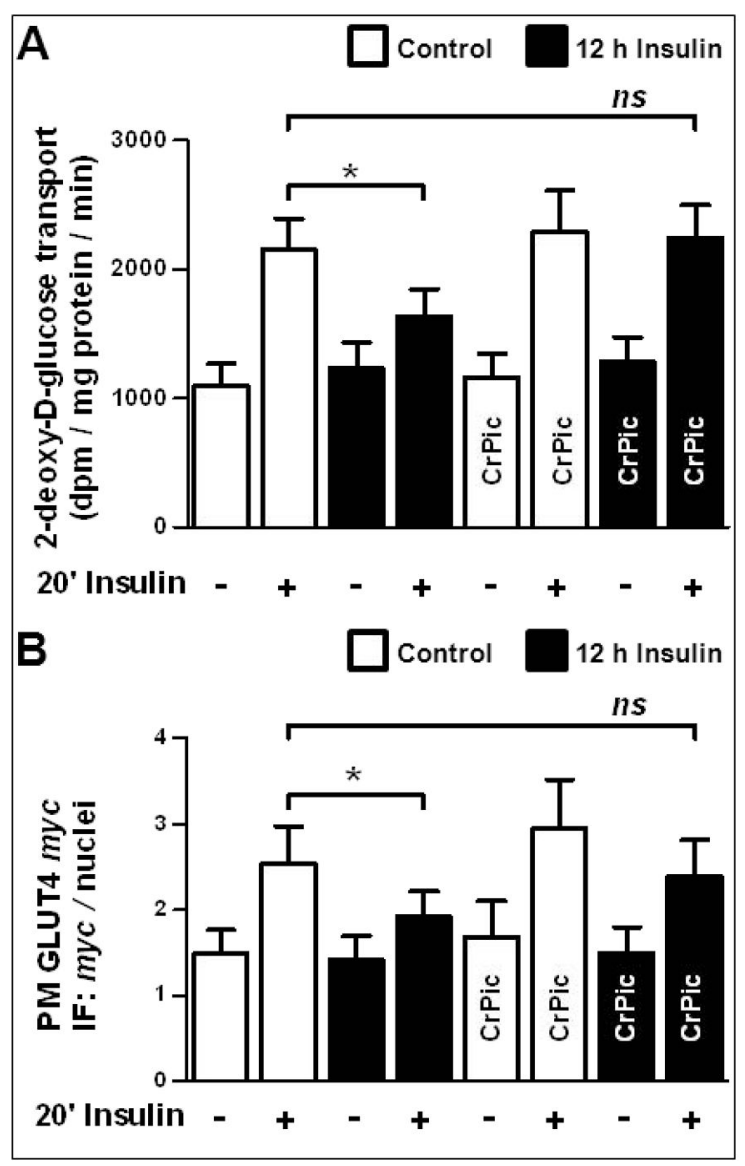

Fig. 1. CrPic protects against hyperinsulinemia-induced GLUT4/glucose transport dysfunction L6-GLUT4myc myotubes were pretreated without (white bars) or with (black bars) $5 \mathrm{nM}$ insulin for $12 \mathrm{~h}$ in the absence or presence of $100 \mathrm{nM} \mathrm{CrPic}$, which was added to the culturing medium $4 \mathrm{~h}$ before the 12-h insulin pretreatment. After these pretreatments, cells were either left untreated (bars 1, 3, 5, and 7) or treated (bars 2, 4, 6, and 8) with $100 \mathrm{nM}$ insulin for $20 \mathrm{~min}$. Immediately following this acute 30-min insulin treatment, 2-deoxy-Dglucose transport (A) and cell surface GLUT4myc immunofluorescence normalized to Syto 60 nuclear stain (B) were determined as described in Materials and Methods. Mean values \pm SEM of are shown from 6-7 independent experiments. All insulin-stimulated transport and GLUT4myc values were significantly $(\mathrm{P}<0.05)$ elevated over their respective controls. *, $\mathrm{P}<0.05$ vs. insulin-stimulated control. 


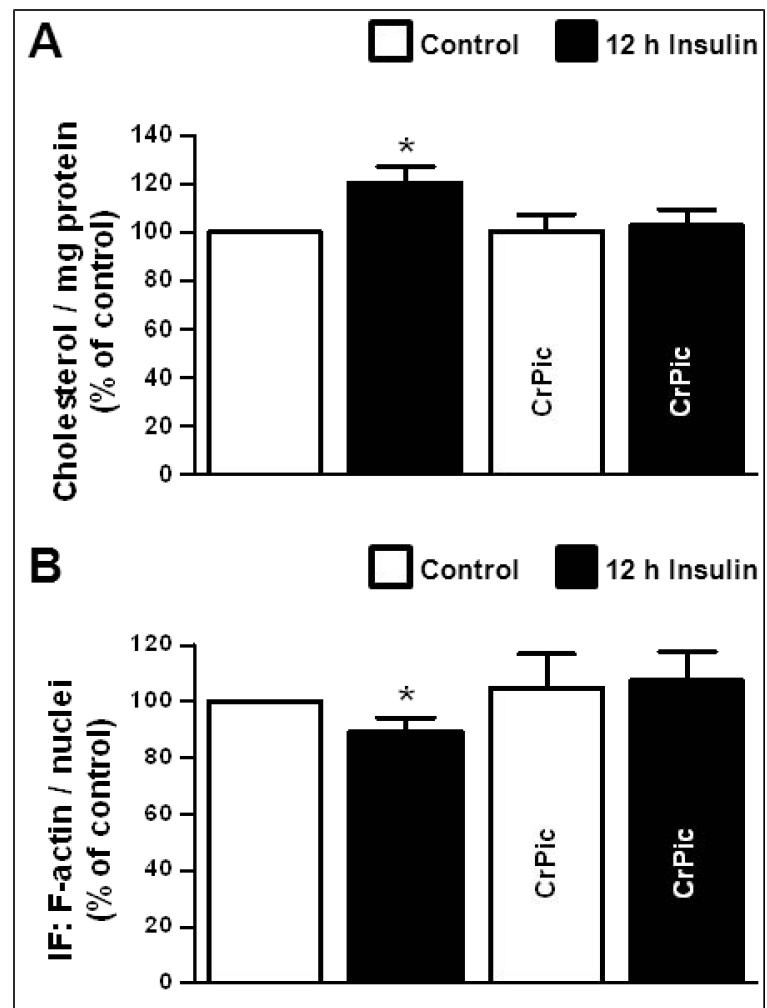

Fig. 2. Hyperinsulinemia-induced membrane cholesterol accumulation and F-actin loss is not present with CrPic pretreatment

Cells were pretreated with or without $\mathrm{CrPic}$ and chronic insulin as described in Fig. 1.

Membrane cholesterol (A) and cortical F-actin (B) content were determined as described in Materials and Methods. Mean values \pm SEM are shown from 4-11 independent experiments. *, $\mathrm{P}<0.05$ vs. control. 




Fig. 3. Insulin signal transduction is not impaired by hyperinsulinemia nor enhanced by CrPic Cells were pretreated with $\mathrm{CrPic}$ and chronic insulin as described in Fig. 1. Cells were then either left untreated (lanes/bars 1, 3, 5, and 7) or treated (lanes/bars 2, 4, 6, and 8) with 100 $\mathrm{nM}$ insulin for $5 \mathrm{~min}$. Immediately following this acute 5-min insulin stimulation, whole-cell lysates were prepared, subjected to SDS/PAGE analysis, and immunoblotted for Akt2 (Ser474) phosphorylation (A), phospho-Akt substrate phosphorylation (B) and $\beta$-actin (C). Note that the representative immunoblot shown for p-AS160 in (B) has been cut between lanes 4 and 5 to align the corresponding treatment groups with the representative immunoblot for total AS160. White and black bars represent control and hyperinsulinemic groups, respectively. Immunoblots are representative of 3-8 experiments. Quantitated values 
are means \pm SEM from those 3-8 separate experiments. All insulin-stimulated values were significantly $(*, \mathrm{P}<0.05)$ elevated over their respective controls. 


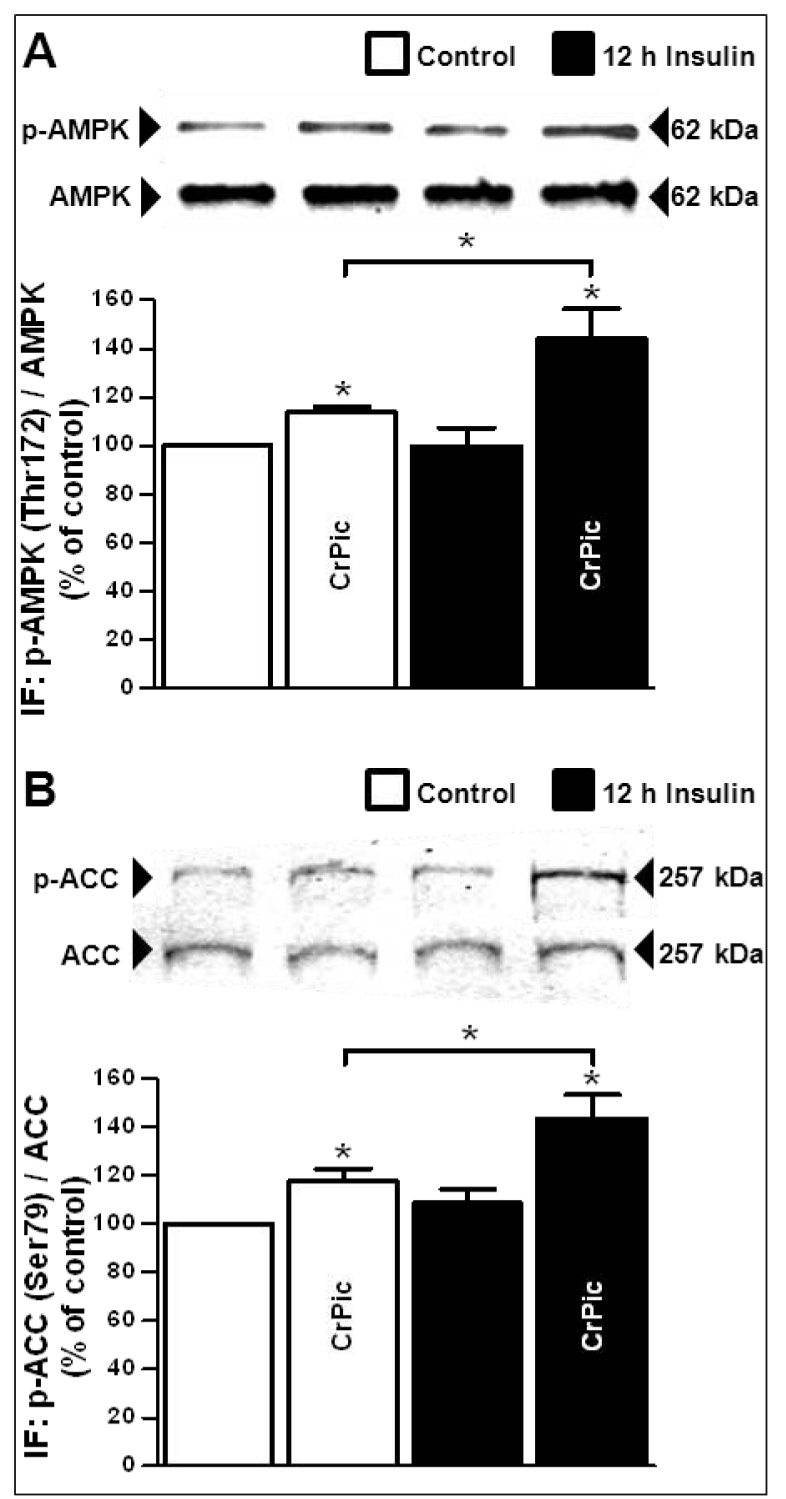

Fig. 4. CrPic increases AMPK activity

Cells were treated with or without $100 \mathrm{nM} \mathrm{CrPic}$ for $16 \mathrm{~h}$ in the absence or presence of $5 \mathrm{nM}$ insulin for the last $12 \mathrm{~h}$. Following these treatments, whole-cell lysates were prepared, subjected to SDS/PAGE analysis, and immunoblotted for AMPK (Thr172) phosphorylation (A) and ACC (Ser79) phosphorylation (B). White and black bars represent control and hyperinsulinemic groups, respectively. Representative immunoblots from 5 separate experiments are shown. Quantitated values normalized to total protein are means $\pm \mathrm{SEM}$ from these 5 independent experiments. *, $\mathrm{P}<0.05$ vs. respective control. 


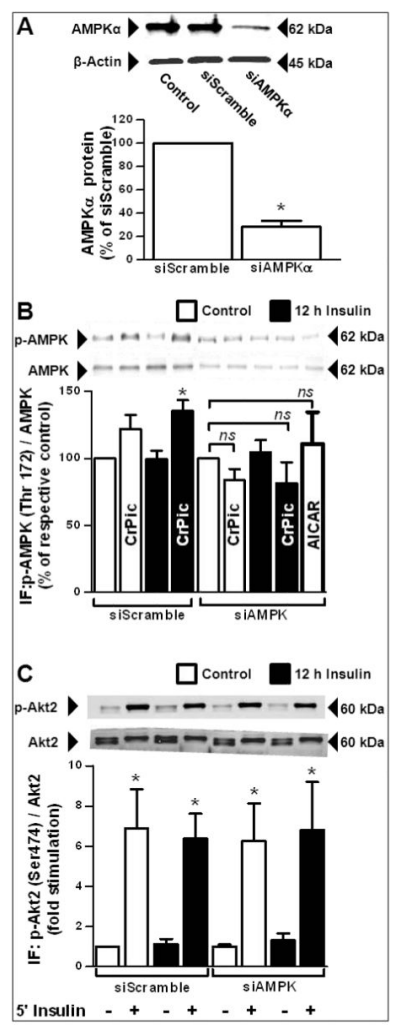

Fig. 5. A substantial loss of the AMPK a-catalytic subunit prevents AMPK activation by CrPic and is not associated with impaired insulin signaling

Following transient siRNA transfection of scrambled control oligos (siScramble) or oligos targeting the a catalytic subunits of AMPK (siAMPK), cells were left untreated, pretreated, and treated as described in preceding legends. Whole-cell lysates were then prepared, subjected to SDS/PAGE analysis, and immunoblotted to assess knockdown efficiency of AMPKa (A), AMPK phosphorylation (B), and Akt2 phosphorylation (C). Representative immunoblots are shown along with quantitated values normalized to $\beta$-actin (A) or total protein (B and $\mathbf{C}$ ) presented as means \pm SEM from 4-5 independent experiments. *, $\mathrm{P}<0.05$ vs siScramble controls. 




Fig. 6. AMPK activity is required for the protective effects of $\mathrm{CrPic}$ against hyperinsulinemiainduced GLUT4 and glucose transport dysfunction

Basal and insulin-stimulated GLUT4myc translocation (A) and 2-deoxy-D-glucose transport (B) were determined in siScramble and siAMPK cells pretreated and treated as described in Fig. 1. Mean fold stimulation values \pm SEM are shown from 6-8 independent experiments. *, $\mathrm{P}<0.05$ vs. insulin-stimulated control. 